\title{
PFP 1: A Large Planetary Nebula Caught in the First Stages of ISM Interaction
}

\author{
Mark J. Pierce A,B,G, David J. Frew ${ }^{\mathrm{B}}$, Quentin A. Parker ${ }^{\mathrm{B}, \mathrm{C}}$, and Joachim Köppen ${ }^{\mathrm{D}-\mathrm{F}}$ \\ A Department of Physics, University of Bristol, Bristol, BS8 1TL, UK \\ B Department of Physics, Macquarie University, Sydney NSW 2109, Australia \\ C Anglo-Australian Observatory, Epping NSW 1710, Australia \\ D Observatoire de Strasbourg, 67000 Strasbourg, France \\ E International Space University, 67400 Illkirch-Graffenstaden, France \\ F Institut für Theoretische Physik und Astrophysik, Universität Kiel, 24098 Kiel, Germany \\ G E-mail: mark.pierce@bristol.ac.uk
}

Received 2004 April 19, accepted 2004 August 2

\begin{abstract}
This paper presents ( $\mathrm{H} \alpha+[\mathrm{NII}])$ imaging and spectroscopy of a previously unknown, highly evolved planetary nebula of low excitation which is in the first stages of an interaction with the interstellar medium (ISM). It was discovered serendipitously from AAO/UKST H $\alpha$ Survey images as part of a project to exploit the survey data and has evaded detection by previous surveys due to its very low surface brightness. It is a remarkable hollow-sphere planetary nebula, some $19^{\prime}$ across, making it one of the largest examples of its type. We estimate a radius of $1.5 \mathrm{pc}$ and a distance of $550 \mathrm{pc}$ as derived from a new $\mathrm{H} \alpha$ surface brightnessradius relation. PFP 1 has near-perfect circular symmetry, broken only at the north-western edge which is coupled with significantly increased $(\mathrm{H} \alpha+[\mathrm{NII}])$ intensity, both of which provide evidence for an interaction with the ISM. We find a near solar composition for this object with possibly enhanced He and $\mathrm{N}$ abundances. A blue central star candidate has been identified from the SuperCosmos Sky Survey data.
\end{abstract}

Keywords: planetary nebulae: general — planetary nebulae: individual (PFP 1) — ISM: abundances — techniques: photometric — techniques: spectroscopic

\section{Introduction}

We report the discovery of a highly evolved, hollow-shell planetary nebula (PN) of large angular size in the early stages of an interaction with the interstellar medium which we designate PFP 1. Old interacting PNe are recognised as valuable probes of the ISM (e.g. Tweedy, Martos, \& Noriega-Crespo 1995; Xilouris et al. 1996; Kerber et al. 2000), but using them to build up a global picture of the ISM properties is hampered by the lack of known examples. This poor detection rate is due to the intrinsic low surface brightness of these objects. The AAO/UKST H $\alpha$ Survey (Parker \& Phillipps 1998, 2003) offers high quality narrowband images for the whole of the Southern Galactic Plane (SGP) and therefore provides a new tool which is uniquely suited to the detection of many more of these large, diffuse PNe.

Because they are so difficult to detect, studies of these objects were relatively neglected until recent years in favour of younger, brighter PNe. Early work by Smith (1976) looked at the effect of a PN-ISM interaction on the position of the central star (CS) with respect to the nebula. Jacoby (1981) was the first to present detailed observational evidence of an interacting PN, Abell 35. However, it was not until later, after analyses by Borkowski, Sarazin, \&
Soker (1990) and Soker, Borkowski, \& Sarazin (1991), that the usefulness of interacting $\mathrm{PNe}$ as probes of the ISM itself was recognised.

These initial studies sparked a renewed effort to find this kind of object. Tweedy \& Kwitter (1996) presented an imaging atlas of the $27 \mathrm{PNe}$ of diameter greater than $8^{\prime}$ known at that time and found evidence for PN-ISM interaction in all but four cases. More recent discoveries have added to the haul (e.g. Rauch 1999; Hewett et al. 2003). However these limited numbers are now being superseded by a significant sample discovered from the new Macquarie/AAO/Strasbourg $\mathrm{H} \alpha \mathrm{PN}$ project (e.g. Parker et al. 2003).

Most previous investigations to find large, faint $\mathrm{PNe}$ have relied on careful scrutiny of broadband photographic survey images, e.g. Melmer \& Weinberger (1990). While it has met with some success, this method is not ideal because the narrow emission lines of PN spectra are diluted in the broad bandpass of the survey filters. This problem is particularly acute for PNe of really low surface brightness and large angular extent. Use of narrowband imaging around an emission line such as $\mathrm{H} \alpha$ alleviates this problem, but has until recently been limited to searches of relatively small areas; e.g. Jacoby \& van de Steene (1995) 
describe a search for new PNe around PG1159 stars using narrowband imaging.

The AAO/UKST H $\alpha$ Survey is now available online (Parker \& Phillipps 2003) and offers an unrivalled means of searching for this kind of low surface brightness object. The survey comprises contemporaneous narrowband $\mathrm{H} \alpha$ and broadband $\mathrm{R}$ photographic images which have been scanned and digitised by the SuperCosmos plate scanning machine at ROE (Hambly et al. 2001) ${ }^{1}$. This SuperCosmos $\mathrm{H} \alpha$ Survey (SHS) is available both as $16 \times$ blockeddown images of each survey field and as full, $0.67^{\prime \prime}$, resolution pixel data. The use of hypersensitised Tech-Pan film (Parker \& Malin 1999) as the detector gives fine, arcsecond spatial resolution and extreme sensitivity to faint diffuse emission (down to $\sim 5 \mathrm{R}^{2}$, Parker et al., in preparation).

The survey data have already been successfully mined for small and moderate-sized PNe through painstaking visual inspection of the survey exposures to yield over 1000 new examples in the Galaxy as a whole (e.g. Parker et al. 2003) as well as over 300 new PNe in the Galactic Bulge (Peyaud, Parker, \& Acker 2003). When completed, this project will more than double the number known in the Southern Hemisphere as compared with the combined work of the previous century.

The serendipitous discovery of PFP 1 from examination of blocked-down $\mathrm{H} \alpha$ data by one of us (M.J.P.) has demonstrated the utility of this technique for discovering large $\left(>4^{\prime}\right)$ emission nebulosities, and has led two of us (D.J.F. and Q.A.P.) to re-examine the entire $\mathrm{H} \alpha$ survey in this way. Rebinning the data to lower resolution enhances the visibility of extended faint nebulosity. Of course, once discovered, the full-resolution pixel data can be examined which permits finer study of the substructure of the PN and any interaction. As a result, more than 30 large PNe candidates were discovered (some up to $30^{\prime}$ diameter), as well as several new optical SNR candidates (cf. Parker, Frew, \& Stupar 2004). Several of the PN candidates have already been spectroscopically confirmed and will be the subject of a separate paper (Frew \& Parker 2004, in preparation).

\section{Observations}

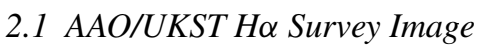

PFP 1 was discovered in the north-western corner of $\mathrm{H} \alpha$ survey field HAL1 109. Basic data pertaining to the object is given in Table 1 and we show a 30' by $30^{\prime}(\mathrm{H} \alpha+[\mathrm{NII}])$ image taken from the SHS data in Figure 1. Most of the limb-brightened nebula is extremely faint, with the brightest part being the north-west rim (see Figure 2). The enhanced limb itself can just be discerned on the POSS I and POSS II red plates and the SERC ER survey as an elongated wisp of nebulosity ( $5^{\prime}$ long) which is easily overlooked; there is no sign of the rest of the $19^{\prime}$ diameter annulus. The wisp is considerably fainter in the blue,

\footnotetext{
${ }^{1}$ www-wfau.roe.ac.uk/sss/halpha/

21 Rayleigh $=10^{6} / 4 \pi$ photons $\mathrm{cm}^{-2} \mathrm{~s}^{-1} \mathrm{sr}^{-1}=$

$2.41 \times 10^{-7} \mathrm{erg} \mathrm{cm}^{-2} \mathrm{~s}^{-1} \mathrm{sr}^{-1}$ at $\mathrm{H} \alpha$.
}

Table 1. Basic data and derived quantities for PFP 1

\begin{tabular}{ll}
\hline Name & PFP 1 \\
\hline Designation & PN G222.1+03.9 \\
$(\alpha, \delta) \mathrm{J} 2000$ & $07^{h} 22^{m} 17.7^{s},-06^{\circ} 21^{\prime} 46.4^{\prime \prime}$ \\
$(l, b)$ & $222.15,3.94$ \\
SHS field & HAL1109 \\
Angular dimensions & $1150^{\prime \prime} \times 1100^{\prime \prime}$ \\
Excitation class & $2^{\mathrm{a}}, 0.6^{\mathrm{b}}$ \\
Reddening, $E(B-V)$ & $0.05 \pm 0.05$ \\
$\log F(\mathrm{H} \alpha)$ & $-10.73 \pm 0.20$ \\
$\log F(\mathrm{H} \beta)$ & $-11.21 \pm 0.21$ \\
Distance & $550 \pm 220 \mathrm{pc}$ \\
Nebular diameter & $3.0 \pm 1.2 \mathrm{pc}$ \\
Nebular mass & $\sim 0.6 \mathrm{M}_{\odot}$ \\
$n_{\mathrm{e}}(\mathrm{rms})$ & $\sim 4 \mathrm{~cm}^{-3}$ \\
Age & $\sim 70 \times 10^{3} \mathrm{yr}$ \\
\hline
\end{tabular}

${ }^{\mathrm{a} A l l e r}$ (1956)

${ }^{\mathrm{b}}$ Dopita \& Meatheringham (1990).

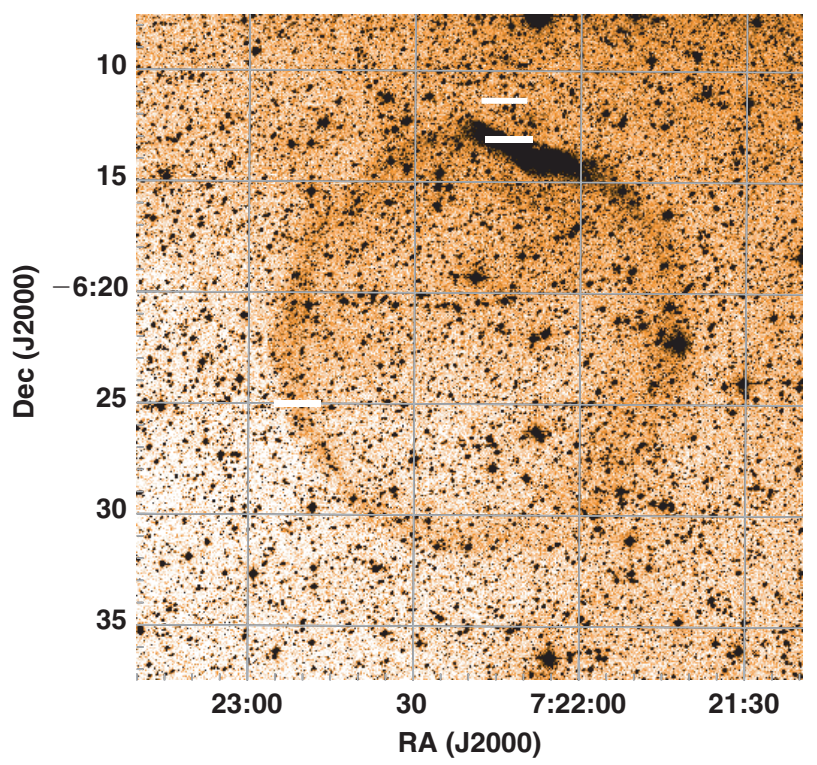

Figure 1 A $30^{\prime}$ by $30^{\prime}$ SHS image of PFP 1 . Several spectra of this object were taken at the SAAO 1.9-m telescope, one of the faint east rim and three of the brightened region as well as an offset sky spectrum. The slit positions are indicated by the white lines.



Figure 2 Full resolution $\sim 8^{\prime}$ by $5^{\prime}$ image of the interacting north-western rim. 
being barely visible at the plate limit on the SERC EJ survey. This is a result of the relatively weak [OIII] emission from this object as revealed by our spectra (Section 2.3). It is obvious from the SHS H $\alpha$ image (Figure 1) that the enhanced rim is concave compared with the rest of the nebula and is the likely signature of an interaction with the ISM. Both the SHS and Southern H $\alpha$ Sky Survey Atlas (SHASSA, Gaustad et al. 2001) $\mathrm{H} \alpha$ images (Figure 7) also show that the eastern rim is somewhat brightened with respect to the south-western edge.

From examination of various online multiwavelength data in the vicinity of PFP 1, such as CO maps (Dame et al. 1987), IRAS dust maps (Wheelock et al. 1994), and the SuperCosmos $\mathrm{H} \alpha$ data itself, it is clear that PFP 1 sits in a region of low extinction, devoid of active star formation. The nearest large scale emission is from the CMa OB1/R1 association which sits $6^{\circ}$ south-west at a distance of $\sim 1400 \mathrm{pc}$ (Mel'Nik \& Efremov 1995). There is also a diffuse $\mathrm{H} \alpha$ filament which extends perpendicular to the plane at $l=225^{\circ}$ (Haffner, Reynolds, \& Tufte 1998) $3^{\circ}$ south of the nebula, which may link with general emission within the Galactic plane.

\subsection{SHASSA and VTSS Images}

The integrated $\mathrm{H} \alpha$ flux for this object was determined from SHASSA data (Gaustad et al. 2001). SHASSA offers narrowband $(\mathrm{H} \alpha+[\mathrm{NII}])$ and continuum $\mathrm{CCD}$ images of the whole southern sky. The data are at much lower spatial resolution ( $48^{\prime \prime}$ pixels) compared with SHS (0.67" pixels) but have the benefit of being continuum-subtracted and intensity-calibrated to a Rayleigh scale. Despite the relatively coarse resolution of the SHASSA data, PFP 1 is such an exceptionally large object that it is easily resolved by the survey.

These continuum-subtracted data were used to measure the flux of both the whole nebula as well as the bright interacting rim. The flux measurement for the whole nebula was taken through a $20^{\prime}$ circular aperture, while the bright region was fitted as tightly as possible with an elliptical aperture. The Starlink routine APERPHOTOM was used in the GAIA image analysis package to make the measurements and the results are given in Table 1 . In order to accurately account for the surrounding diffuse $\mathrm{H} \alpha$ emission, measurements of sky background were made through an aperture identical to the object aperture at fifteen representative regions immediately surrounding the nebula. The scatter in these is our principal uncertainty in the flux measurement.

The continuum-subtracted SHASSA data come in two forms: raw, $48^{\prime \prime}$ resolution or data smoothed to $4^{\prime}$. The full-resolution SHASSA data shows some unphysical, negative pixel values which are residuals from poorly subtracted stellar images. To check that these do not unduly influence the flux measurement, we carried out the same procedure on SHASSA fields for 40 wellstudied PN (of a range of surface brightness) with an independent measure of $\mathrm{H} \alpha$ flux given in the literature. This confirmed that, despite the artefacts, aperture photometry on the full resolution data returns the best measurement of the $\mathrm{H} \alpha$ flux. Results agree with published data to $\Delta F(\mathrm{H} \alpha)=-0.001 \mathrm{dex}, \sigma=0.07$ for SHASSA minus literature fluxes.

The $[\mathrm{NII}] / \mathrm{H} \alpha$ ratios for the north-western enhancement and the rest of the limb-brightened sphere (from the spectra described in Section 2.3) were used to deconvolve the $\mathrm{H} \alpha$ and [NII] contributions from the SHASSA red flux, knowing the bandpass of the filter (Gaustad et al. 2001). Their quoted zero-point error of $9 \%$ and our estimated error in the integrated $[\mathrm{NII}] / \mathrm{H} \alpha$ ratio for the whole nebula of $30 \%$, have been added quadratically to get the overall uncertainty in the flux. We estimate $\log F(\mathrm{H} \alpha)=-10.73 \pm 0.20$ and use the observed $\mathrm{H} \alpha / \mathrm{H} \beta$ ratio (from our spectra) to derive $\log F(\mathrm{H} \beta)=$ $-11.21 \pm 0.21$.

We also looked at the relevant field (Mon07) from the Virginia Tech Spectral-Line Survey (VTSS; Dennison, Simonetti, \& Topasna 1998). The VTSS resolution is coarser than SHASSA (1.6' pixels) and consequently it is more difficult to adequately subtract the diffuse background. On the other hand, the $\mathrm{H} \alpha$ filter has a narrower bandpass and the [NII] contribution can be ignored. Gaustad et al. (2001) found the VTSS data to be fainter than SHASSA by a factor of 1.25 in one field, while Finkbeiner (2003) found this offset to be applicable to all fields in common. We confirm these findings by estimating $\mathrm{H} \alpha$ fluxes from VTSS for nine well-studied PNe on different fields, finding an offset of 0.09 dex (VTSS fainter). We applied this correction to the integrated flux for PFP 1 estimated from the VTSS image and obtained $\log F(\mathrm{H} \alpha)=-10.8 \pm 0.3$, where the error is dominated by the uncertain background subtraction. Nevertheless, this is in excellent agreement with our [NII]-corrected flux derived from SHASSA.

\subsection{Spectroscopic Observations}

Spectra were obtained with the SAAO 1.9-m telescope on the nights of 2004 Feb 13 and Feb 14. The standard grating spectrograph was used with a SITe $1798 \times 266$ pixel CCD coupled to an $f / 2.2$ camera with an $86-\mathrm{mm}$ beam. Grating 7 was used (300 lines $\mathrm{mm}^{-1}$ ), giving a spectral coverage of 3600-7400 $\AA$. On-chip binning $(2 \times)$ perpendicular to the dispersion direction was used to reduce readout noise. The slit width was widened to $400 \mu \mathrm{m}$ ( $\sim 2.4^{\prime \prime}$ on the sky) to allow greater throughput for the faint emission of the extended $\mathrm{PNe}$ observed on this run, though with some attendent loss of resolution (to $\sim 7 \AA$ ). The unvignetted slit length perpendicular to the dispersion was $1.6^{\prime}$ and was fixed in an east-west direction. Spectra of both the faint eastern edge of the PN shell and the bright rim were obtained in separate observations summarised in Table 2. An offset sky frame was also taken as the nebula subtended the entire slit. The positions of the slit are shown in Figure 1. Dome screen flats from a quartz-iodine lamp and twilight flats were also obtained together with observations of spectrophotometric standard stars (Stone \& Baldwin 1983) and PN flux standards 
(Dopita \& Hua 1997). Wavelength calibration was applied via $\mathrm{Cu}-\mathrm{Ar}$ arc exposures bracketing the observations. The spectra were reduced and examined using standard IRAF routines.

The two spectra of the north-western rim taken on Feb 14 were combined and used for the analysis which follows. As the conditions on Feb 13 were not photometric, the spectrum taken on that night was examined separately and confirms the findings from the two combined spectra. The combined north-western spectrum is plotted in Figure 3.

Table 3 includes the measured flux values, normalised to $\mathrm{H} \beta=100$ in column 3 and the fluxes corrected for reddening in column 4, for $\mathrm{H} \alpha / \mathrm{H} \beta=2.85$ (assuming $T_{\mathrm{e}}=10^{4}$, Brocklehurst 1971) and adopting the $R=3.1$ reddening law of Savage \& Mathis (1979). The intensities of the brighter lines are estimated to be accurate to $30 \%$ (50\% for the lines marked with a colon), and as an internal check, the ratios [OIII] $\lambda 5007 / \lambda 4959=2.8$ and $[\mathrm{NII}] \lambda 6584 / \lambda 6548=3.2$, agree well with the theoretical values of each. We derive the logarithmic extinction at

Table 2. Log of spectroscopic observations

\begin{tabular}{lll}
\hline $\begin{array}{l}\text { Date } \\
\text { (UT) }\end{array}$ & \multicolumn{1}{c}{$\begin{array}{c}\text { Slit position } \\
(\alpha, \delta) \mathrm{J} 2000\end{array}$} & $\begin{array}{l}\text { Exposure } \\
(\mathrm{s})\end{array}$ \\
\hline 2004 Feb 13 & $\begin{array}{l}\text { north-western rim: } \\
07^{h} 22^{m} 13^{s},-06^{\circ} 13^{\prime} 05^{\prime \prime}\end{array}$ & 1200 \\
2004 Feb 14 & $\begin{array}{l}\text { north-western rim: } \\
07^{h} 22^{m} 13^{s},-06^{\circ} 13^{\prime} 05^{\prime \prime}\end{array}$ & $900 \times 2$ \\
2004 Feb 14 & $\begin{array}{l}\text { eastern rim: } \\
07^{h} 22^{m} 51^{s},-06^{\circ} 25^{\prime} 00^{\prime \prime}\end{array}$ & 1200 \\
2004 Feb 14 & $\begin{array}{l}07^{h} 22^{m} 14^{s},-06^{\circ} 11^{\prime} 20^{\prime \prime} \\
\end{array}$ & 900 \\
\hline
\end{tabular}

$\mathrm{H} \beta, c=0.07$, which leads to $E(B-V)=0.05$ and a total visual absorption, $A_{V}=0.15$.

\subsection{Identification of Candidate Central Star}

In order to find potential central star candidates, we examined digitised UKST $R$ and $B_{\mathrm{J}}$ plates from the SuperCosmos Sky Survey (hereafter SSS; Hambly et al.

Table 3. Line fluxes and ratios for PFP 1 from the co-added spectra of the interacting north-west rim

\begin{tabular}{lcrc}
\hline ID & Wavelength $(\AA)$ & $F(\lambda)$ & $I(\lambda)$ \\
\hline$[\mathrm{OII}]$ & 3727 & $585:$ & $611:$ \\
{$[\mathrm{NeIII}]$} & 3869 & $77:$ & $80:$ \\
$\mathrm{H} \gamma$ & 4340 & 45 & 46 \\
$\mathrm{H} \beta$ & 4861 & 100 & 100 \\
{$[\mathrm{OIII}]$} & 4959 & 50 & 50 \\
{$[\mathrm{OIII}]$} & 5007 & 141 & 140 \\
{$[\mathrm{NI}]$} & 5199 & $29:$ & $29:$ \\
$\mathrm{HeI}$ & 5876 & $18:$ & $18:$ \\
{$[\mathrm{OI}]$} & $6300^{\mathrm{A}}$ & $108:$ & $103:$ \\
{$[\mathrm{OI}]$} & $6364^{\mathrm{A}}$ & $35:$ & $33:$ \\
{$[\mathrm{NII}]$} & $6548^{\mathrm{B}}$ & 228 & 216 \\
$\mathrm{H} \alpha$ & $6563^{\mathrm{B}}$ & 301 & 285 \\
{$[\mathrm{NII}]$} & $6584^{\mathrm{B}}$ & 727 & 688 \\
{$[\mathrm{SII}]$} & $6717^{\mathrm{B}}$ & 133 & 125 \\
{$[\mathrm{SII}]$} & $6731^{\mathrm{B}}$ & 85 & 80 \\
{$[\mathrm{NII}] / \mathrm{H} \alpha$} & & & $3.17 \pm 0.20$ \\
{$[\mathrm{OIII}] / \mathrm{H} \beta$} & & & $1.90 \pm 0.10$ \\
{$[\mathrm{SII}] 6717 / 6731$} & & & $1.57 \pm 0.05$ \\
\hline
\end{tabular}

$F(\mathrm{H} \beta)=1.96 \times 10^{-14} \mathrm{erg} \mathrm{cm}^{-2} \mathrm{~s}^{-1}, c(\mathrm{H} \beta)=0.07$.

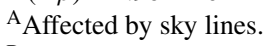

${ }^{\mathrm{B}}$ From Gaussian deblends of close lines.

: Denotes higher uncertainty.



Figure 3 The combined, flux calibrated spectrum of the north-western rim. 



Figure 4 Candidate central star at $07^{h} 22^{m} 17.7^{s},-06^{\circ} 21^{\prime} 46.4^{\prime \prime}$ in UKST $B_{\mathrm{J}}$ (left) and $R$ band (right) images. Each image is $3^{\prime}$ on a side. The candidate CS is highlighted in each case by the arrow.
Table 4. Magnitudes and colours for the candidate central star which sits at the geometric centre of the nebula. $B_{\mathrm{J}}$ magnitude is estimated from the SuperCosmos Sky Survey data as described in Section 2.4 and $R_{2}$ indicates the second epoch $R$-band survey

\begin{tabular}{lll}
\hline Band & Source & Magnitude \\
\hline$B_{\mathrm{J}}$ & SSS & $15.43 \pm 0.15$ \\
$R_{2}$ & SSS & $15.45 \pm 0.15$ \\
$I$ & SSS & $16.51 \pm 0.15$ \\
$J$ & 2 MASS & $16.64 \pm 0.16$ \\
Colour & & \\
$\quad B_{\mathrm{J}}-R$ & & -0.02 \\
$\quad R-I$ & & -1.06 \\
$B_{\mathrm{J}}-J$ & & -1.21 \\
\hline
\end{tabular}

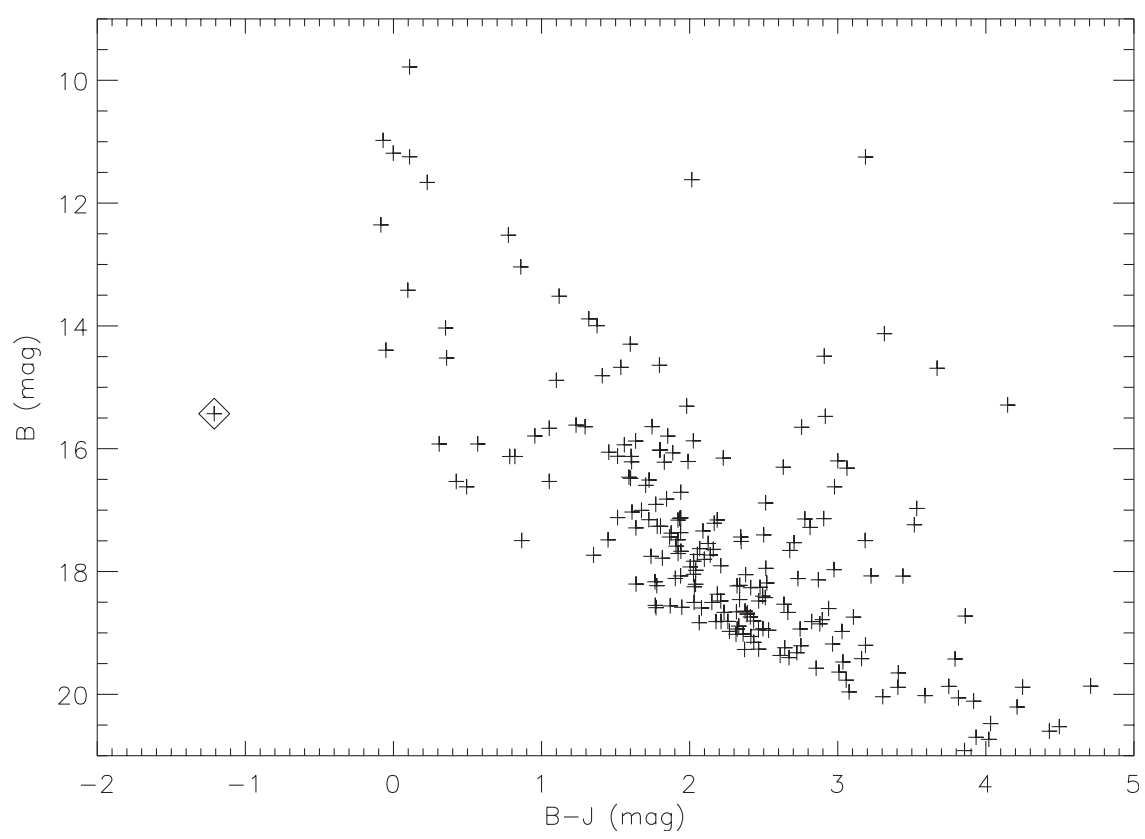

Figure $5 B_{\mathrm{J}}$ vs $B_{\mathrm{J}}-J$ colour-magnitude diagram for objects within $10^{\prime}$ of the geometric centre of the nebula, using SSS $B_{\mathrm{J}}$ and 2MASS $J$ magnitudes (see text). The CSPN candidate is highlighted with a diamond and lies well to the blue of the disk main sequence.

$2001)^{3}$. A careful visual inspection revealed an object just $15^{\prime \prime}$ from the geometric centre of this large nebula which is brighter in the blue plate than the red when compared with other stars in the field. The two images of this object are shown in Figure 4, first in $B_{\mathrm{J}}$ then in $R$. North is up and east is left.

Some additional work was required to determine the $B_{\mathrm{J}}$ magnitude from the SSS data. The standard SuperCosmos detection and parameterisation software had not deblended the candidate CS from its nearest neighbour to the east in the blue data, although this pair were separated in $R$ as both components are fainter in that bandpass. We used the Starlink routine SEXTRACTOR in conjunction with GAIA to independently deblend these two objects from the UKST $B_{\mathrm{J}}$ pixel data. As SEXTRACTOR provides a flux measurement from aperture photometry for each object, we were able to make flux measurements for $\sim 2000$ stars within $10^{\prime}$ of our candidate CS. This formed

\footnotetext{
3 www-wfau.roe.ac.uk/s.s / index.html
}

a well-constrained $B_{\mathrm{J}}$-flux relation which could be used to calibrate the $B_{\mathrm{J}}$ magnitude of the now deblended CS candidate based on its measured flux. Fitting to the calibration curve in this way gave us a magnitude estimate of $B_{\mathrm{J}} \sim 15.43 \pm 0.15 \mathrm{mag}$ for our favoured CSPN. This magnitude is consistent with those tabulated in the USNO-B1.0 and GSC 2.2.01 surveys which use the same plate material.

In Table 4 we show photometric measurements of the candidate CS obtained from the SSS, as well as the $J$ $(1.6 \mu \mathrm{m})$ magnitude from the Two Micron All Sky Survey (2MASS) Point Source Catalog (Cutri et al. 2003). Along with the blue $B_{\mathrm{J}}-R$ colour of $\sim 0.0$, the $B_{\mathrm{J}}-J$ colour of -1.2 indicates that this is a very blue object and is consistent with the predicted $B_{\mathrm{J}}-J=-1.0$ for a $100000 \mathrm{~K} \mathrm{CSPN}$ (see below), giving us confidence that we have indeed identified the true central star. This is further confirmed by the star's position in the $B_{\mathrm{J}}$ vs $B_{\mathrm{J}}-J$ colour-magnitude diagram in Figure 5.

An apparent blue magnitude of $\sim 15.4$ is quite a reasonable value for an evolved CSPN at the distance and 
reddening that we derive for PFP 1 . The calculated $M_{B_{\mathrm{J}}} \simeq 6.6_{-0.9}^{+1.1}$ is similar to the central star of the large interacting PN Sh 2-216, which has $M_{B}=6.5$ from an accurately determined trigonometric parallax (Harris et al. 1997). In addition, we use the hydrogen-burning tracks of Blöcker (1995) to estimate the luminosity of 0.605 and $0.625 \mathrm{M}_{\odot} \mathrm{CSPN}$ at an age of 70000 years (cf. Sections 3.1 and 3.2). Blöcker's tracks predict a temperature of $\sim 100000 \mathrm{~K}$ and a luminosity of $50-60 \mathrm{~L}_{\odot}$. Using the mean of the bolometric corrections from Schönberner (1981), Cahn (1984) and Mendez et al. (1988), quoted by Cazetta \& Maciel (1994), we calculate $M_{B}=6.6-6.9$, which is also in good agreement with our SHS magnitude estimate.

Furthermore, if the nebula were an ionisation-bounded Strömgren sphere with volume filling factor, $\epsilon=0.3$, at a distance of $550 \mathrm{pc}$ (cf. Section 3.1), the observed $\mathrm{H} \beta$ flux would require $1.8 \times 10^{45}$ ionising photons $\mathrm{sec}^{-1}$ from the central star. With an assumed temperature of $100000 \mathrm{~K}$ its luminosity would be $25 \mathrm{~L}_{\odot}$. It could be higher, since the nebula may be density bounded. But one has to keep in mind that a nebula of such a low density will have a recombination time of the order of 100000 years and thus the present state of ionisation does not reflect the current state of the central star, but rather its state some time ago (cf. Kerber et al. 1999). At any rate, it seems clear that the central star is well on the low-luminosity part of its evolutionary track and on the way to becoming a white dwarf. This ties in well with the highly evolved state of the nebula.

\section{Analysis}

\subsection{Distance and Mass}

In the absence of definitive photometric and spectroscopic information on the candidate central star, the distance can be estimated by the classical Shklovsky method, assuming a value for the ionised mass of the shell. Inverting this procedure allows the determination of ionised masses for a number of evolved PNe of similar evolutionary age and morphology to our object, with distances taken from the literature. Ionised masses were calculated using the formula of Pottasch (1996):

$$
M=4.03 \times 10^{-4} \epsilon^{1 / 2} d^{5 / 2}(\mathrm{H} \beta)^{1 / 2} \Theta^{3 / 2} \mathrm{M}_{\odot}
$$

where $d$ is the distance $[\mathrm{kpc}], \mathrm{H} \beta$ is the flux $\left[10^{-11} \mathrm{erg}\right.$ $\mathrm{cm}^{-2} \mathrm{~s}^{-1}$ ], and $\Theta$ is the nebular radius [arcseconds]. We have used a volume filling factor, $\epsilon=0.3$ (cf. Section 3.2 and Mallik \& Peimbert 1988; Boffi \& Stanghellini 1994; Pottasch 1996). The range of ionised masses for 25 low surface brightness PNe with good distance determinations is 0.06 to $1.3 \mathrm{M}_{\odot}$, excluding the anomalous objects PHL 932 (Mendez et al. 1988; Acker et al. 1998) and K 1-27 (Rauch, Köppen, \& Werner 1994; Ciardullo et al. 1999). This brackets the distance of PFP 1 to between 240 and 770 pc.

For highly evolved PNe, we can make use of the fact that the central star fades as the nebula evolves.
Furthermore, since the most massive PNe have optically fainter central stars, the difference between the central star magnitude and the integrated $\mathrm{H} \beta$ magnitude of the nebula is an indicator of the ionised mass of the object, and functions as a distance-independent parameter. We have found for low surface brightness PNe with $\log S(\mathrm{H} \beta) \leq$ $-3.5 \mathrm{erg} \mathrm{cm}^{-2} \mathrm{~s}^{-1} \mathrm{sr}^{-1}$ that $\Delta=m_{B, \mathrm{CS}}-m_{\mathrm{H} \beta \text {, neb ranges }}$ between -4.0 and +4.0 and correlates quite well with ionised mass (Frew \& Parker, in preparation). The $\mathrm{H} \beta$ magnitudes were derived using the formula of Jacoby (1989) and are corrected for reddening. High excitation, optically thick PNe (such as NGC 246 \& Abell 36; Kaler 1981) have $\Delta \leq-1.0$ and are found to have quite low masses (below $0.12 \mathrm{M}_{\odot}$ ), but several old low-excitation nebulae (e.g. Abell 21, Sh 2-188, Sh 2-216) with $\Delta>0$ are considerably more massive (from 0.3 up to at least $1.3 \mathrm{M}_{\odot}$ ). PFP 1 , with $\Delta=+1.2$, thus has a relatively faint central star and its excitation class is low (see Section 3.2). Therefore it most likely has a moderately high ionised mass, larger than the mean of $0.3 \mathrm{M}_{\odot}$ for evolved PNe. It follows that the distance is unlikely to be near the lower bound of our bracketed range.

An alternative distance method is to construct an empirical H $\alpha$ surface brightness-radius relation, calibrated with $\mathrm{PNe}$ which have accurate distance estimates determined using a primary method. A preliminary version of this new clear relation is shown in Figure 6. We note that it is broadly consistent with an $R^{-3}$ law as found for Magellanic Cloud PNe by Stanghellini et al. (2003). From our estimated dereddened $\log S(\mathrm{H} \alpha)=-6.05$ for PFP 1 , we derive a radius of $1.5 \pm 0.6 \mathrm{pc}$, and hence a distance of $550 \pm 220 \mathrm{pc}$. The estimated ionised mass is $0.6 \mathrm{M}_{\odot}$ (for an assumed $\epsilon=0.3$ ) which is comfortably within the range found for evolved low-excitation $\mathrm{PNe}$. We intend to improve our distance estimate by obtaining accurate UBV photometry and a spectrum of the CSPN candidate.

\subsection{Other Nebular Parameters}

We used the combined spectrum of the north-western rim to estimate the principal nebular parameters, tabulated in Table 1. We were also able to measure an $[\mathrm{NII}] / \mathrm{H} \alpha$ ratio in the much fainter eastern rim for comparison purposes (see Section 4). The derived reddening, $E(B-V)=0.05$, can be compared to the asymptotic extinction of $E(B-V)=0.26\left(A_{V}=0.81\right)$ in this direction, as determined from the exinction map of Schlegel, Finkbeiner, \& Davis (1998). This is an unusually transparent sightline through the disk, despite the low galactic latitude $\left(b=3.9^{\circ}\right)$, so no accurate distance estimate is possible using the extinction-distance method.

The surface brightness at $\mathrm{H} \alpha$ derived from the brightest 71 binned pixels ( 99 arcsec) along the slit is $\log S(\mathrm{H} \alpha)=-4.97$, which can be compared with $\log S(\mathrm{H} \alpha)=-5.43$ derived using the brightest SHASSA pixel value, after deconvolving the [NII] contribution to the SHASSA data and correcting for background. There is a $30 \%$ probable error on the $\mathrm{H} \alpha$ flux through the slit which sampled the brightest portion of the north-western 


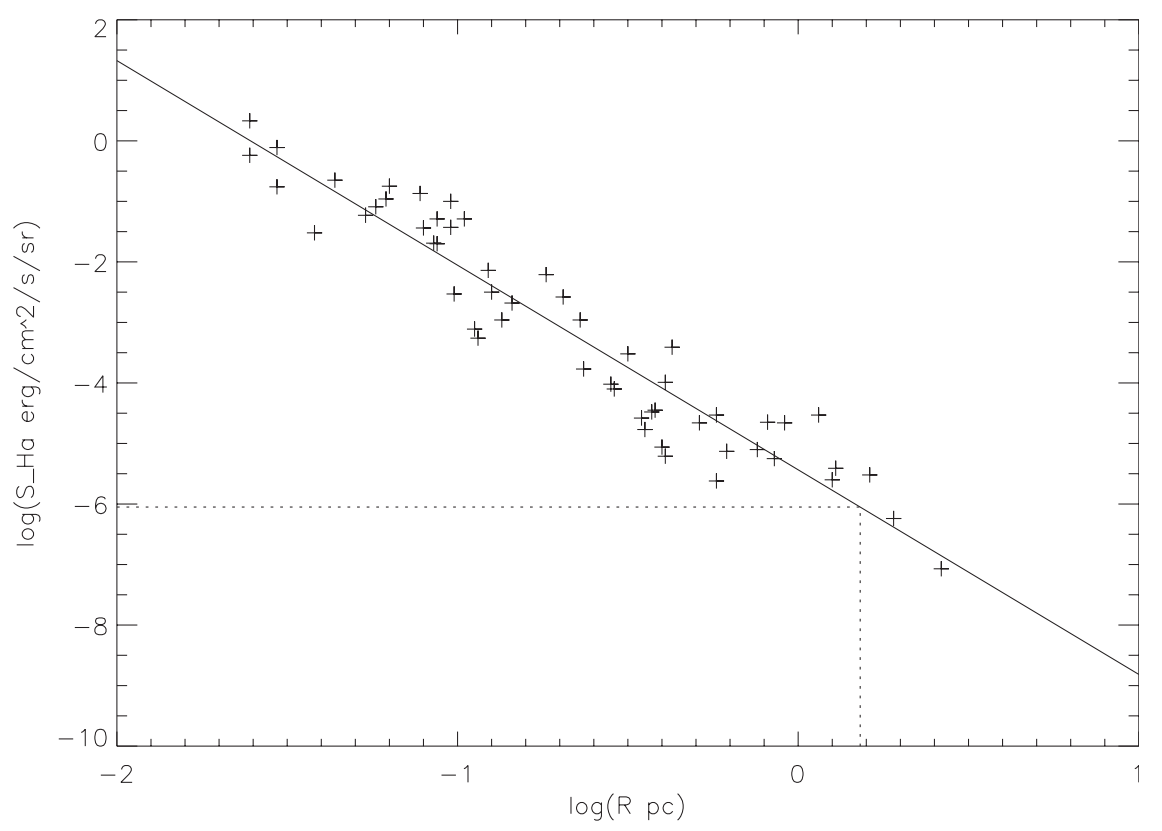

Figure 6 An empirical, dereddened $\mathrm{H} \alpha$ surface brightness-radius relation for planetary nebulae based on 53 local calibrators with well-determined distances selected from the literature (Frew \& Parker, in preparation). The calibrating PNe range in brightness from young objects like NGC 7027 through to faint examples similar to our new PN. The best fit is represented by $\log S(\mathrm{H} \alpha)=-5.43-3.38 \times \log R$ and is consistent with an $R^{-3}$ law. The position of PFP 1 is indicated by the dashed lines.

rim, which is only about $35^{\prime \prime}$ north to south. Considering that each $48^{\prime \prime}$ SHASSA pixel in the vicinity would sample nebulosity of varying surface brightness, we consider these values to be in reasonable agreement. The interacting rim is therefore a factor of five to ten higher in surface brightness than that averaged over the entire nebula.

The centre-to-limb variation of the surface brightness from a thin spherical shell is characterised by a brightened rim. As the surface brightness of an optically thin nebula is proportional to the pathlength through the nebula, the ratio of the maximum brightness and the central brightness is

$$
I_{\max } / I_{\text {centre }}=\frac{\sqrt{R^{2}-(R-\delta)^{2}}}{\delta} \approx \sqrt{\frac{2 R}{\delta}}
$$

for an outer radius $R$ and shell thickness $\delta$.

From the image in Figure 1 we can directly estimate the shell thickness as $\delta / R \approx 0.1$. This gives an average intensity ratio of 4.5 between rim and the centre, consistent with the observed ratio between just the bright interacting portion of the rim and the whole nebula. This also gives a volume filling factor of $1-((R-\delta) / R)^{3}=0.3$, if we assume the interior to be completely empty.

The excitation class is low, only 2 on the scale of Aller (1956), as revealed by the relatively strong [NII] and [OII] lines and weak [OIII] emission seen in our spectra. The spectrum shows very strong similarity to the bright eastern section of Sh 2-216 (Fesen, Blair, \& Gull 1981), though the [NII] emission is somewhat stronger; this may be an abundance effect. A decimal excitation class of 0.6 was calculated according to the scheme of Dopita \& Meatheringham (1990). Both indices indicate a nebula of low excitation, as expected for a highly evolved PN of large intrinsic diameter surrounding a faint evolved central star on the white dwarf cooling track.

We are unable to calculate the electron temperature due to the non-detection of the [OIII] $\lambda 4363$ and the $[\mathrm{NII}] \lambda 5755$ lines which are typically weaker than the $[\mathrm{O} I I] \lambda 5007$ lines by a large factor. From the [SII] $\lambda 6717 /$ 6731 ratio of 1.57 we can only find the limit for $n_{\mathrm{e}}<10^{2} \mathrm{~cm}^{-3}$ (Stanghellini \& Kaler 1989) because the [SII] ratio is insensitive to electron density at this value. We can also calculate an approximate value of $n_{\mathrm{e}}$ from the observed $\mathrm{H} \alpha$ flux, radius, and distance of the object according to the relation:

$$
n_{\mathrm{e}}^{2}=2.8 \times 10^{22} \frac{F(H \alpha)}{\epsilon \Theta^{3} D},
$$

where $\epsilon$ is the filling factor, $\Theta$ is the radius [arcsec], and $D$ is the distance [pc]. We get $n_{\mathrm{e}} \sim 4 \mathrm{~cm}^{-3}$, which is one of the lowest values known. Making simple assumptions, we roughly estimate that the density in the bright rim (where we observed the spectrum) is about three times higher.

The kinematic age can be crudely estimated assuming an expansion velocity of $20 \mathrm{~km} \mathrm{~s}^{-1}$ (Borkowski et al. 1990). The age is then $\sim 70000 \mathrm{yr}$ although this is a possible lower limit because the expansion rate is likely to have slowed over the course of its evolution.

Although we only have low dispersion spectra, using the IRAF EMSAO emission line fitting routines in the RVSAO package leads to a heliocentric radial velocity of $-18 \pm 19 \mathrm{~km} \mathrm{~s}^{-1}$, based on fits to eight lines. Comparing equivalent measures for two Dopita \& Hua (1997) PNe standards observed on the same night, we find agreement to $\pm 20 \mathrm{~km} \mathrm{~s}^{-1}$. 
Table 5. Ionic and elemental abundances of PFP 1. The elemental abundances are given in the usual form of $12+\log [n(\mathrm{X}) / n(\mathrm{H})]$

\begin{tabular}{ll}
\hline$n\left(\mathrm{He}^{+}\right) / n\left(\mathrm{H}^{+}\right)$ & 0.13 \\
$n\left(\mathrm{~N}^{0}\right) / n\left(\mathrm{H}^{+}\right)$ & $1.30 \times 10^{-5}$ \\
$n\left(\mathrm{~N}^{+}\right) / n\left(\mathrm{H}^{+}\right)$ & $1.32 \times 10^{-4}$ \\
$n\left(\mathrm{O}^{0}\right) / n\left(\mathrm{H}^{+}\right)$ & $1.92 \times 10^{-4}$ \\
$n\left(\mathrm{O}^{+}\right) / n\left(\mathrm{H}^{+}\right)$ & $2.27 \times 10^{-4}$ \\
$n\left(\mathrm{O}^{++}\right) / n\left(\mathrm{H}^{+}\right)$ & $5.26 \times 10^{-5}$ \\
$n\left(\mathrm{Ne}^{++}\right) / n\left(\mathrm{H}^{+}\right)$ & $8.08 \times 10^{-5}$ \\
$n\left(\mathrm{~S}^{+}\right) / n\left(\mathrm{H}^{+}\right)$ & $4.71 \times 10^{-6}$ \\
$\mathrm{He}$ & 11.11 \\
$\mathrm{~N}$ & 8.44 \\
$\mathrm{O}$ & 8.67 \\
$\mathrm{Ne}$ & 8.86 \\
$\mathrm{~S}$ & 6.31 \\
\hline
\end{tabular}

\subsection{Abundance Ratios}

We conducted a preliminary interpretation of the nebular spectrum, using the plasma diagnostics program HOPPLA (for a description see Acker et al. 1989; Köppen, Acker, \& Stenholm 1991). Since no line ratio for the electron temperature is available, we assume a constant value of $10^{4} \mathrm{~K}$. The [SII] doublet ratio is in the low density limit, and we have used a density of $12 \mathrm{~cm}^{-3}$ for the north-western rim (see Section 3.2). It should be noted that at such a low density, none of the lines are sensitive to collisional deexcitation, so the derived abundances are not dependent on the assumed value of $n_{\mathrm{e}}$. The results are collected in Table 5. Helium appears to be somewhat enhanced but one should keep in mind that the HeI $\lambda 5876$ is close to the noise level of the spectrum. Oxygen is nearly solar with neutral oxygen lines contributing about $40 \%$. The N/O ratio is -0.23 dex, just above the minimum value of -0.3 dex for a Type I PN according to Peimbert \& Torres-Peimbert (1983), but less than the cutoff of -0.1 dex proposed by Kingsburgh \& Barlow (1994). Furthermore, the simple morphology (a probable hollow-sphere) is not typical of a Type I nebula.

Though the quality of the available spectra do not allow more than a first estimate of the elemental abundances, we can deduce that the chemical composition of this PN is close to solar. There is a possiblility that helium and nitrogen may be somewhat enhanced, but we can exclude that this nebula is an extreme Type I object. This is corroborated by locating the object in the $\mathrm{H} \alpha /[\mathrm{NII}]$ vs $\mathrm{H} \alpha /[\mathrm{SII}]$ diagnostic diagram (e.g. Tajitsu et al. 1999). With $\log (\mathrm{H} \alpha /[\mathrm{NII}])=-0.38$ and $\log (\mathrm{H} \alpha /[\mathrm{SII}])=+0.14$, it resembles photoionisation models of low density $\mathrm{PNe}$ around low temperature and low luminosity central stars, and is close to the domain of supernova remnants.

\section{ISM Interaction}

Tweedy \& Kwitter (1996) suggest three criteria to confirm that PN asymmetry is caused by a PN-ISM interaction.
They propose that such interactions are characterised by an asymmetry in the outer region of the nebula, accompanied by an increase in brightness at the interacting edge which also exhibits an increased $[\mathrm{NII}] / \mathrm{H} \alpha$ ratio with respect to the rest of the nebula. The high $[\mathrm{NII}] / \mathrm{H} \alpha$ ratio results from material being compressed at the PN-ISM boundary, raising the density which, in turn, increases the recombination rate and lowers the ionisation state of that portion of gas (see also Kerber et al. 2000). The new PN presented in this paper can be seen to satisfy the first two criteria solely from the SHS image. The beginning of an asymmetry, coupled with an increase in brightness, can be clearly seen in Figure 1. Our spectroscopic measurements of line ratios (see Table 3) satisfy the third criterion where the bright rim does have an enhanced $[\mathrm{NII}] / \mathrm{H} \alpha$ ratio of 3.2 compared with the east rim of the nebula where $[\mathrm{NII}] / \mathrm{H} \alpha=1.5$. The ratio for the whole nebula is $[\mathrm{NII}] / \mathrm{H} \alpha=1.8$.

That the interaction is at an early stage is illustrated by the position of the CS, which is still close to the centre of PFP 1; only the north-west rim is brightened, implying that the bulk of the nebula is still moving with the CS. Objects showing more advanced interaction may have a substantially displaced CS, e.g. Sh 2-174 (Tweedy \& Napiwotzki 1994) where the CS has actually left the nebula, and the extreme case of Sh 2-216 (Tweedy et al. 1995) where the CS is displaced by $24^{\prime}$ from the geometric centre of this $1.6^{\circ}$ diameter $\mathrm{PN}$, due to the nebular material being slowed and ultimately reabsorbed into the ISM.

Borkowski et al. (1990) discuss PN-ISM interactions in terms of the expansion velocity of the PN and the velocity of the progenitor star with respect to its surroundings. In a homogeneous, isotropic ISM, the PN will start to interact on its leading edge. As the PN expansion causes the nebular density to fall below a critical value, the gas in the shell is compressed, resulting in a brightness enhancement in this direction. As the interaction develops the leading edge of the nebula is slowed by the ISM and the PN shell becomes distorted as we observe in the case of PFP 1 where an opposite concave curvature is seen.

At first glance this appears to be a reasonable explanation for the morphology of the new PN, especially as our examination of the local Galactic environment found that it sits in a low density region despite its small $z$ distance of $40 \mathrm{pc}$. We could therefore conclude that the interaction is taking place because the $\mathrm{PN}$ is moving with respect to the local ISM, implying that the CS is moving north across the field.

As an alternative cause of the interaction, we also investigated whether the PN is simply expanding into a region or cloud of enhanced density to the north-west. An IRAS $100-\mu \mathrm{m}$ dust map does show very faint dust emission sitting to the south of PFP 1 with a prominence that curls round the west edge and extends to the interacting rim. The IRAS data are plotted in Figure 7 as contours over the SHASSA $\mathrm{H} \alpha$ image. In the absence of distance information for the emitting dust we cannot tell whether this really is responsible for the PN interaction or if it is merely a line-of-sight coincidence. 


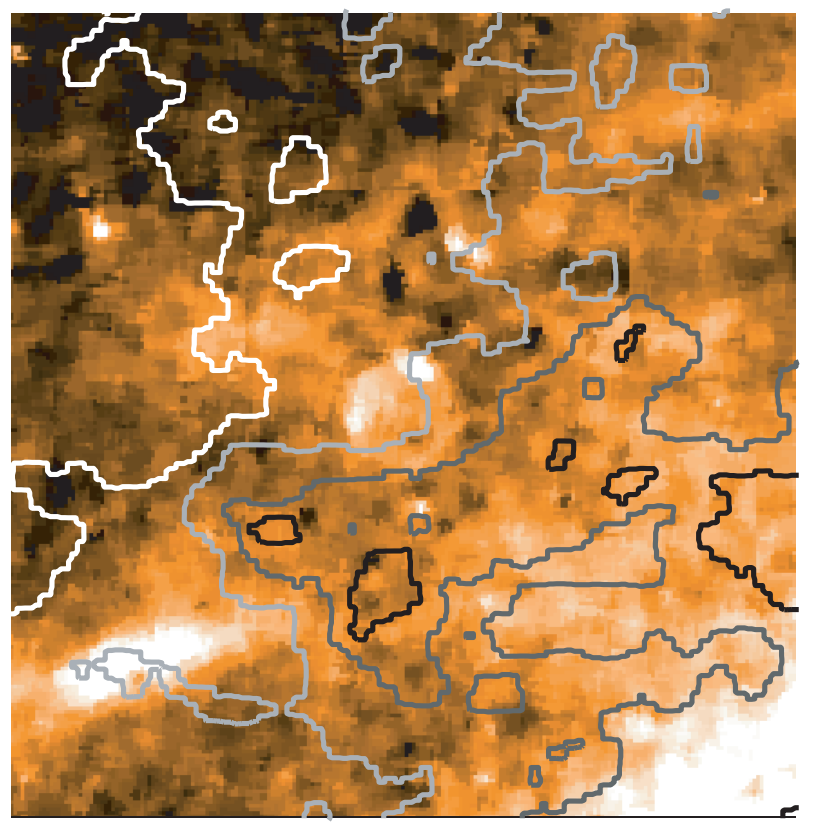

Figure 7 IRAS $100 \mu \mathrm{m}$ contours overlaid on a $\sim 2^{\circ}$ by $2^{\circ}$ area of smoothed SHASSA H $\alpha$ data. The IRAS contours are at 10 (white), 12,14 , and 16 (black) $\mathrm{MJy} \mathrm{sr}^{-1}$.

\section{Summary}

We present preliminary observational findings of an extremely large, highly coherent, ring-shaped planetary nebula which has evidence for preliminary interaction with the local ISM. Using a new $\mathrm{H} \alpha$ surface brightnessradius relation we derive a distance of $\sim 550 \mathrm{pc}$ for the new PN which is consistent with limits obtained from the inverted Shlkovsky method. This distance, coupled with the estimated $\epsilon=0.3$ based on the PN geometry, gives a nebular mass of $0.6 \mathrm{M}_{\odot}$ which is consistent with other highly evolved PNe. A good candidate CS has been identified from UKST $B_{\mathrm{J}}$ and $R$ plates. Follow up spectroscopy is planned to confirm whether this is the true central star. The lack of adjacent $\mathrm{CO}$ and IRAS dust emission explains why PFP 1 has been able to expand unencumbered to its present size of $\sim 3.0 \mathrm{pc}$. We find two possible causes for the interaction evident on the north-western rim - the motion of the PN with respect to its surroundings as per Borkowski et al. (1990) or an encounter with a region of enhanced density as it expands. We are unable to state conclusively which is responsible based on the available data. An accurate proper motion determination of the CS candidate would be useful in establishing which of these scenarios is most likely. Analysis of moderate resolution spectra has shown that this is a PN of low excitation, which is consistent with other large-diameter senile examples. Although the spectra have low signal-to-noise, we find the chemical composition of this $\mathrm{PN}$ is probably close to solar, and while it exhibits a high $[\mathrm{NII}] / \mathrm{H} \alpha$ ratio it is doubtful whether it is a bona fide Type I PN as defined by Kingsburgh \& Barlow (1994).

The same technique which led to the discovery of PFP 1 has furnished us with $\sim 30$ other candidate $\mathrm{PNe}$ of size $>4^{\prime}$. A follow up program is underway to obtain spectra and narrowband imaging of this sample.

\section{Acknowledgments}

This study used data from the AAO/UKST H $\alpha$ Survey, now available online as the SuperCosmos $\mathrm{H} \alpha$ Survey (SHS) by the WFAU of the Royal Observatory, Edinburgh. These data were supplemented with calibrated images from the Southern H $\alpha$ Sky Survey Atlas (SHASSA) and data from the Virginia Tech Spectral-Line Survey (VTSS) which were produced with support from the National Science Foundation. This research has made use of the SIMBAD database, operated at CDS, Strasbourg, France. This publication makes use of data products from the Two Micron All Sky Survey (2MASS), which is a joint project of the University of Massachusetts and the Infrared Processing and Analysis Center/California Institute of Technology, funded by the National Aeronautics and Space Administration and the National Science Foundation. M.J.P. acknowledges support from PPARC and D.J.F. acknowledges an Australian Postgraduate Award and an ANSTO travel grant. Q.A.P. acknowledges support from the AAO and Macquarie University.

\section{References}

Acker, A., Fresneau, A., Pottasch, S. R., \& Jasniewicz, G. 1998, A\&A, 337, 253

Acker, A., Jasniewicz, G., Köppen, J., \& Stenholm, B. 1989, A\&AS, 80,201

Aller, L. H. 1956, Gaseous Nebulae (London: Chapman \& Hall)

Blöcker, T. 1995, A\&A, 299, 755

Boffi, F. R., \& Stanghellini, L. 1994, A\&A, 284, 248

Borkowski, K. J., Sarazin, C. L., \& Soker, N. 1990, ApJ, 360, 173

Brocklehurst, M. 1971, MNRAS, 153, 471

Cahn, J. H. 1984, ApJ, 279, 304

Cazetta, J. O., \& Maciel, W. J. 1994, A\&A, 290, 936

Ciardullo, R., Bond, H. E., Sipior, M. S., Fullton, L. K., Zhang, C.-Y., \& Schaefer, K. G. 1999, AJ, 118, 488

Cutri, R. M., Skrutskie, M. F., van Dyk, S., Beichman, C. A., Carpenter, J. M., Chester, T., Cambresy, L., Evans, T., et al. 2003, VizieR Online Data Catalog, 2246

Dame, T. M., Ungerechts, H., Cohen, R. S., de Geus, E. J., Grenier, I. A., May, J., Murphy, D. C., Nyman, L.-A., et al. 1987, ApJ, 322, 706

Dennison, B., Simonetti, J. H., \& Topasna, G. A. 1998, PASA, 15,147

Dopita, M. A., \& Hua, C. T. 1997, ApJS, 108, 515

Dopita, M. A., \& Meatheringham, S. J. 1990, ApJ, 357, 140

Fesen, R. A., Blair, W. P., \& Gull, T. R. 1981, ApJ, 245, 131

Finkbeiner, D. P. 2003, ApJS, 146, 407

Gaustad, J. E., McCullough, P. R., Rosing, W., \& Van Buren, D. 2001, PASP, 113, 1326

Haffner, L. M., Reynolds, R. J., \& Tufte, S. L. 1998, ApJ, 501, L83

Hambly, N. C., MacGillivray, H. T., Read, M. A., Tritton, S. B., Thomson, E. B., Kelly, B. D., Morgan, D. H., Smith, R. E., et al. 2001, MNRAS, 326, 1279

Harris, H. C., Dahn, C. C., Monet, D. G., \& Pier, J. R. 1997, in IAU Symp. 180: Planetary Nebulae, 40

Hewett, P. C., Irwin, M. J., Skillman, E. D., Foltz, C. B., Willis, J. P., Warren, S. J., \& Walton, N. A. 2003, ApJ, 599, L37

Jacoby, G. H. 1981, ApJ, 244, 903

Jacoby, G. H. 1989, ApJ, 339, 39

Jacoby, G. H., \& van de Steene, G. 1995, AJ, 110, 1285

Kaler, J. B. 1981, ApJ, 250, L31 
Kerber, F., Furlan, E., Rauch, T., \& Roth, M. 2000, in ASP Conf. Ser. 199: Asymmetrical Planetary Nebulae II: From Origins to Microstructures, 313

Kerber, F., Köppen, J., Roth, M., \& Trager, S. C. 1999, A\&A, 344, L79

Kingsburgh, R. L., \& Barlow, M. J. 1994, MNRAS, 271, 257

Köppen, J., Acker, A., \& Stenholm, B. 1991, A\&A, 248, 197

Mallik, D. C. V., \& Peimbert, M. 1988, RMxAA, 16, 111

Melmer, D., \& Weinberger, R. 1990, MNRAS, 243, 236

Mel'Nik, A. M., \& Efremov, Y. N. 1995, AstL, 21, 10

Mendez, R. H., Kudritzki, R. P., Groth, H. G., Husfeld, D., \& Herrero, A. 1988, A\&A, 197, L25

Parker, Q. A., Frew, D. J., \& Stupar, M. 2004, AAONw, 104, 9

Parker, Q. A., \& Malin, D. 1999, PASA, 16, 288

Parker, Q. A., \& Phillipps, S. 1998, PASA, 15, 28

Parker, Q. A., \& Phillipps, S. 2003, in ASP Conf. Ser. 289: Proceedings of the IAU 8th Asian-Pacific Regional Meeting, Vol I, 165-176

Parker, Q. A., et al. 2003, in IAU Symp. 209: Planetary Nebulae and their Role in the Universe, 25

Peimbert, M., \& Torres-Peimbert, S. 1983, in IAU Symp. 103: Planetary Nebulae, 233-241

Peyaud, A. E. J., Parker, Q. A., \& Acker, A. 2003, in SF2A-2003: Semaine de l'Astrophysique Francaise, 311
Pottasch, S. R. 1996, A\&A, 307, 561

Rauch, T. 1999, A\&AS, 135, 487

Rauch, T., Köppen, J., \& Werner, K. 1994, A\&A, 286, 543

Savage, B. D., \& Mathis, J. S. 1979, ARA\&A, 17, 73

Schlegel, D. J., Finkbeiner, D. P., \& Davis, M. 1998, ApJ, 500, 525

Schönberner, D. 1981, A\&A, 103, 119

Smith, H. 1976, MNRAS, 175, 419

Soker, N., Borkowski, K. J., \& Sarazin, C. L. 1991, AJ, 102, 1381

Stanghellini, L., \& Kaler, J. B. 1989, ApJ, 343, 811

Stanghellini, L., Shaw, R. A., Balick, B., Mutchler, M., Blades, J. C., \& Villaver, E. 2003, ApJ, 596, 997

Stone, R. P. S., \& Baldwin, J. A. 1983, MNRAS, 204, 347

Tajitsu, A., Tamura, S., Yadoumaru, Y., Weinberger, R., \& Köppen, J. 1999, PASP, 111, 1157

Tweedy, R. W., \& Kwitter, K. B. 1996, ApJS, 107, 255

Tweedy, R. W., Martos, M. A., \& Noriega-Crespo, A. 1995, ApJ, 447, 257

Tweedy, R. W., \& Napiwotzki, R. 1994, AJ, 108, 978

Wheelock, S. L., Gautier, T. N., Chillemi, J., Kester, D., McCallon, H., Oken, C., White, J., Gregorich, D., et al. 1994, NASA STI/Recon Tech Report N, 95, 22539

Xilouris, K. M., Papamastorakis, J., Paleologou, E., \& Terzian, Y. 1996, A\&A, 310, 603 